\title{
Computer Vision Techniques for Cancerous Cells Analysis in FIB-SEM Images
}

Guillaume Thibault $^{1 *}$, Jessica Riesterer ${ }^{1,3}$, Kevin Stoltz ${ }^{1}$, Kevin Loftis ${ }^{1}$, Geoffrey Schau ${ }^{1,2}$, Erin Stempinski $^{1,3}$, Claudia López ${ }^{1,3}$, Young Hwan Chang ${ }^{1,2}$ and Joe W. Gray ${ }^{1}$

1. OHSU Center for System Spatial Biomedicine (OCSSB), Biomedical Engineering, Portland OR, USA.

2. Computational Biology Program, OHSU), Portland OR, USA.

3. Multiscale Microscopy Core, Oregon Health \& Science University (OHSU), Portland OR, USA.

* Corresponding author: thibaulg@ohsu.edu

Recent advances in focused ion beam-scanning electron microscopy (FIB-SEM) has led to unprecedent visualization, analysis, and thus understanding of cancerous cells structures, as well as cell-to-cell interaction in cancerous tissues [1]. If a typical dataset contains hundreds of images (usually up to 1400 images of $6 \mathrm{Kx} 4 \mathrm{~K}$ pixels with $4 \mathrm{~nm}$ resolution per pixel) allowing a $3 \mathrm{D}$ reconstruction of a tissue fraction, it is not ready for analysis at it is because of the following issues: $1 /$ misalignment, each consecutive two images are not properly aligned and need registration to preserve the $3 \mathrm{D}$ information continuity; 2/ noise, depending on the acquisition settings, the images may contain an important quantity of noise making any further analysis tedious or even impossible; $3 /$ the structures of interest touch each other or even interlaced, making visualization and spatial analysis impossible.

The first step to process the FIB-SEM images is to perform a registration. To do so, an in-house stochastic version of TurboReg [2] is used: the feature points selection and matching is performed several times and the median transformation matrix is computed to perform the final registration. This stochastic approach can handle highly noisy dataset where local artifact, feature points within resin area, or tissues with sparse population of cells complicate the identification of reliable features point detection. An empirical assessment over the past years of experiments has shown that our stochastic approach fixes any type of error that may occur and no misalignment had been reported.

The second step is image denoising to improve the signal and visually highlight the structures of interest. Deep learning approaches can be adopted for such tasks: If a training dataset can be built by collecting the same image many times before slicing with the FIB, a ground truth image can be computed by taking each pixel median value across each scan, and then use a deep learning architecture as described in [3]; Else, a variational autoencoder architecture [4] should be preferred as the noise is a random signal added to the real information and cannot be numerically modelized. Therefore, when using an autoencoder the encoder part will encode the signal while excluding the random noise, which behaves as an image denoiser. Figure 1 shows the result of the architecture presented in [3].

The final step is to segment the cell structures of interest to visualize them in $3 \mathrm{D}$ in their microenvironment, but manual annotation of the entire dataset is time and labor intensive (up to months), and thus an automatic or at least semi-automatic method is required. Here, we focus on two structures to segment with deep learning, the nuclei and nucleoli. Unfortunately, since morphological and texture features of cancerous tissues are so heterogeneous even intra-dataset, it is not possible to perform transfer learning, i.e. re-train a pre-trained model with few datasets and apply it on new unseen datasets. However, it is feasible to manually segment few images sequentially or manually selected throughout a dataset, train a model on these selected images, and then propagate the information on the unselected images. This approach has shown promising results [5] (see figure 2), reducing the manual segmentation 
burden by a factor of 20 [6].

\section{References:}

[1] CJ.Peddie and LM Collinson, Micron 61 (2014), p. 9.

[2] P Thévenaz, UE Ruttimann and M Unser, IEEE Transactions on Image Processing 7(1) (1998), p. 27.

[3] T Remez et al., "Deep Convolutional Denoising of Low-Light Images", arXiv:1701.01687 (2017).

[4] DP Kingma and M Welling, "Auto-Encoding Variational Bayes”, arXiv:1312.6114 (2013).

[5] K Loftis et al., QBI (2019).

[6] Electron microscopy was performed at the Multiscale Microscopy Core (MMC) with technical support from the Oregon Health \& Science University (OHSU)-FEI Living Lab and the OHSU Center for Spatial Systems Biomedicine (OCSSB). The authors acknowledge funding from the OHSU BrendenColson Center for Pancreatic Care, the Prospect Creek Foundation and the OCSSB.

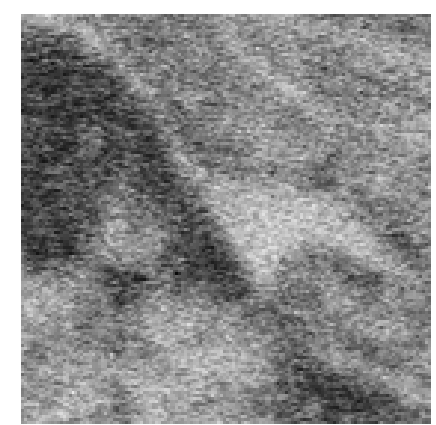

Input

$\mathrm{MAE}=14.49$

$\mathrm{MSE}=346.6$

$\mathrm{PSNR}=22.79$

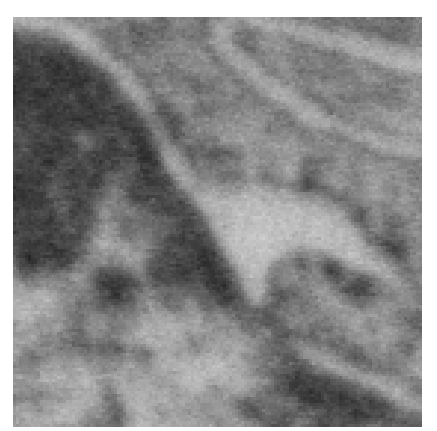

Ground Truth

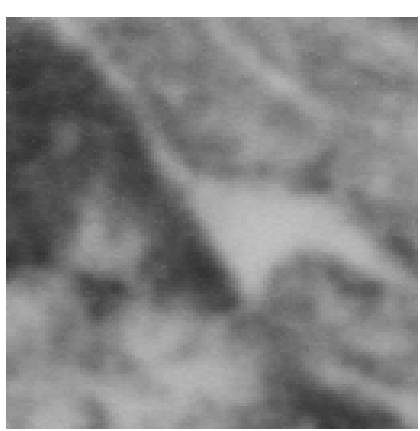

Output

$$
\begin{aligned}
\text { MAE } & =6.98 \\
\text { MSE } & =80.6 \\
\text { PSNR } & =29.19
\end{aligned}
$$

Figure 1. Denoising results using the architecture described in [3] with the following measures: Mean Absolute Error (MAE), Mean Square Error (MSE) and Peak Signal to Noise Ratio (PSNR).
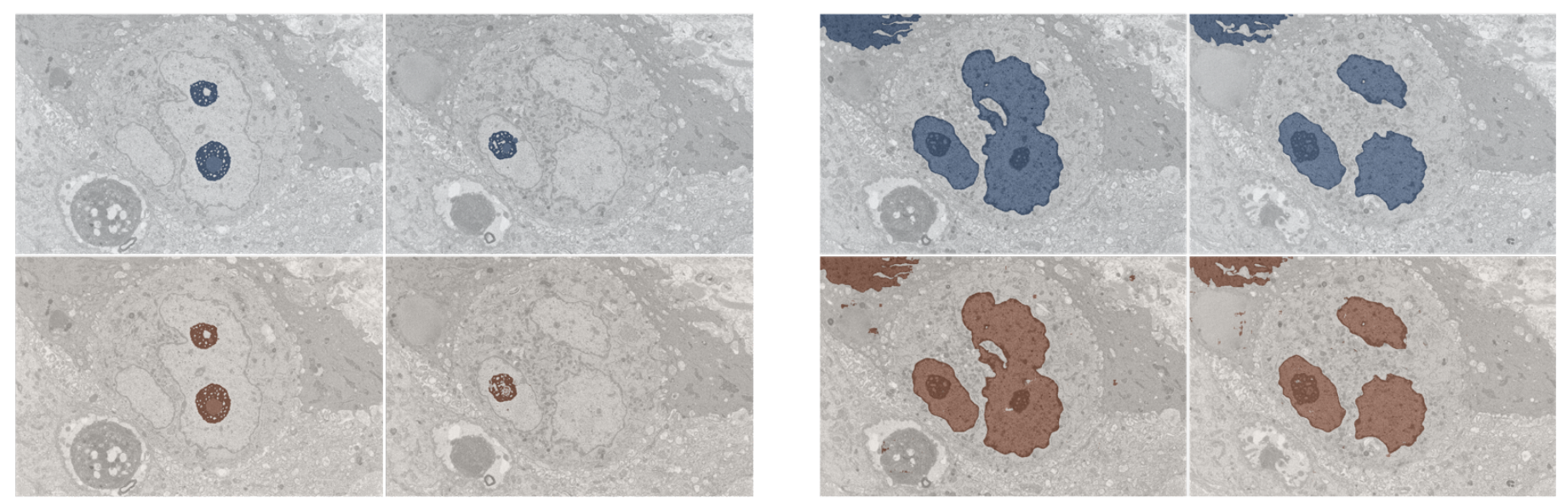

Figure 2. Nucleoli (left) and nuclei (right) segmentation results for two different datasets: the ground truth is in blue and the segmentation in red. 